\title{
Coupling Independent Operation in Wireless Power Transfer System without Ferrite Usage
}

\author{
Nattapong Hatchavanich ${ }^{1, *}$, Sumate Naetiladdanon ${ }^{2}$, Anawach Sangswang ${ }^{3}$, Mongkol Konghirun ${ }^{4}$ \\ Department of Electrical Engineering, King Mongkut's University of Technology Thonburi, Thailand \\ ${ }^{1}$ Email: nattapong.hat@kmutt.ac.th \\ ${ }^{2}$ Email:sumate.nae@kmutt.ac.th \\ ${ }^{3}$ Email: anawach.san@kmutt.ac.th \\ ${ }^{4}$ Email: mongkon.kon@kmutt.ac.th \\ * Corresponding author
}

\begin{abstract}
The power transfer efficiency and output power of a wireless power transfer (WPT) system are mainly affected by magnetic coupling between the primary and secondary coils. This paper presents a constant-current series-series compensated WPT system. Based on the bifurcation criteria, $k_{\text {cri }}$ and $L_{c r i}$, the splitting zero phase angle (ZPA) frequencies is adopted as the operating frequency. The proposed system remains fully compensated even under coupling variations, and without ferrite. The current and voltage gains at the operating frequency can be estimated through the primary current and voltage. A phase-locked loop circuit is used to track the corresponding ZPA frequency due to the coil positioning variations. Experimental results have shown that the 1-kW of output power with the satisfied efficiency of $96 \%$.
\end{abstract}

Keywords: Bifurcation, Coupling Independent, WPT.

\section{INTRODUCTION}

Wireless power transfer (WPT) systems have been widely used for transferring the power through air gap. The magnetic coupling between the primary and secondary coils can significantly alter the power transfer capability and efficiency. A reactive power compensation is commonly chosen to overcome the issue of poor magnetic coupling due to large leakage flux. Typical topologies are series-series (SS), series-parallel (SP), parallel-parallel (PP) and parallelseries (PS) [1]. Advantages and disadvantages of each topology have been reported [2] and [3]. For a parallel compensation, the primary side is modelled as an equivalent current source, which requires an additional component to transfer energy from a voltage source [4] and [5]. On the other hand, the primary side of the series resonant compensation is modelled as a voltage source. The seriesseries (SS) topology is the commonly used among other resonant compensation topologies, due to its simplicity and high system efficiency. In addition, the operating frequency is rather constant even under coupling variations [6]. The high-order compensation such as LCC-LCL can improve the overall system efficiency and the current limit under misalignment condition [7]

The coupling coefficient is an important influence on the output power and the system efficiency. The coupling is highly dependent with the area through where the mutual magnetic flux passes. A larger vertical distance or horizontal misalignment requires larger coil dimension. Conventional techniques in improving the magnetic coupling involve ferrite core usage, installed beneath the coils [8]. The main drawbacks of ferrite inclusion are cost, weight, and brittle property, especially in EV applications. A misalignment between the coils frequently occur in practice since most EVs are manually operated by driver. The coil misalignment strongly decreases the coupling and leads to efficiency reduction. An inclusion of the third coil has shown to improve the misalignment tolerance up to $40 \%$ [9]. Relatively recently, a new coil type with better coupling coefficient than the DD and circular flat configurations in misalignment position has been proposed [10]. In the process of coil improvement, simplicity and cost are often traded off for better coupling.

Normally, the SS compensation circuit is used with a voltage source inverter (VSI). The SS compensation, under light load or poor magnetic coupling, has only one zero phase angle (ZPA) operating point at the resonant frequency. As either the load or the magnetic coupling increases, the splitting ZPA frequencies appear [11]. Once the bifurcation occurs, regardless of compensation type, the resonant frequency no longer offers the maximum output power. A controller with frequency tracking control for ZVS operation as in [12] may be operated in non ZVS mode since the impedance characteristics have been changed and damaged due to hard switching are unavoidable. Most WPT systems are designed to avoid the bifurcated region because a sudden change of impedance characteristics after the bifurcation boundary is undesirable. In [13], the quality factor on the secondary circuit is adjusted to ensure a bifurcation-free operation. The critical coupling $\left(k_{c r i}\right)$ introduced in [14] suggests that the coupling should be less than $k_{c r i}$ to avoid the bifurcation.

As the demand for better performance and efficiency increases, recent efforts have been directed toward developing WPT systems with radio frequency (RF) range operation. In [15], the magnetically coupled resonator has shown a potential to wirelessly deliver power efficiently. The coupling $k_{c r i}$ for output power regulation with nearconstant efficiency corresponding to the greatest distance is proposed. The frequency splitting occurs at $k_{c r i}$ and the transfer efficiency can be maintained regardless of the distance provided that the appropriate frequency is tracked. So far, the application is limited in low-power applications. In this paper, we extend the analysis to take advantage of the magnetically coupled resonator and apply to the inductively coupled WPT system without ferrite. Moreover, the multi- 
primary coils are applied to the primary side to improve the misalignment tolerance. A part of the investigation is devoted to rationalizing the commonly observed bifurcation. New ZPA operating frequencies in the bifurcated region of the SS-WPT system are proposed with the aim for constant power and constant efficiency under coupling variation. Necessary conditions for the proposed operation are investigated. With the proposed method, the current gains remain constant throughout the coupling and load variations. The inherent benefit includes a removal of voltage and current sensors on the secondary circuit since the secondary voltage and current can be directly estimated through the primary voltage and current. With the proposed variable frequency controller, the output power and efficiency remain constant under practical load and coupling variations.

\section{WPT SYSTEM DESCRIPTIONS}

A WPT system is typically designed to deliver power to the load over a relatively large air gap. The structure of the WPT system can be depicted in Fig. 1. The resonant circuit supplies high alternating current to produce sufficient magnetic flux. A typical SS-WPT system is illustrated as the coupled inductor model, as shown in Fig. 2. The voltage source inverter is supplied by the input voltage $\left(V_{i n}\right)$ to the resonant network. The primary capacitance $\left(C_{l}\right)$ is resonated with the primary coil inductance $\left(L_{1}\right)$. Likewise, the secondary capacitance $\left(C_{2}\right)$ is resonated with the secondary coil inductance $\left(L_{2}\right)$. The primary and secondary circuits are magnetically linked by the mutual inductance $(M)$. At the resonant frequency, only the resistive load is reflected to the primary circuit. A power converter is often connected to the secondary circuit. The converter on the secondary circuit can supply both inductive and capacitive loads. Without loss of generality, the load is modelled as an equivalent load resistance $\left(R_{L}\right)$, since the inductive or capacitive effect of the load can essentially be reflected in the secondary circuit parameters. Both primary and secondary circuits are tuned to operate at the natural frequency $\left(\omega_{0}\right)$ which can be expressed as,

$$
\omega_{0}=1 / \sqrt{L_{1} C_{1}}=1 / \sqrt{L_{2} C_{2}}
$$

Thus, the primary capacitance is given as,

$$
\begin{gathered}
C_{1}=L_{2} C_{2} / L_{1} \quad \omega_{2}=\sqrt{\frac{C_{2}^{2}\left(R_{L}\right)^{2}-\frac{2}{\omega_{0}^{2}}+\sqrt{\frac{4}{\omega_{0}^{4}}\left(k_{12}^{2}-1\right)+\left[\frac{2}{\omega_{0}^{2}}-C^{2}\right.}}{\frac{2}{\omega_{0}^{4}}\left(k_{12}^{2}-1\right)}} \\
\operatorname{Re}\left\{Z_{\text {in }}\right\}=\frac{\left(L_{1} L_{2} R_{L} C_{2}^{2} k^{2} \omega^{4}\right)}{\left(1+C_{2}\left(C_{2} R_{L}^{2}-2 L_{2}\right) \omega^{2}+\left(C_{2} L_{2}\right)^{2} \omega^{4}\right)} \\
\operatorname{Im}\left\{Z_{\text {in }}\right\}=-\frac{L_{1}\left(1+C_{2}\left(C_{2} R_{L}^{2}-3 L_{2}\right) \omega^{2}+L_{2} C_{2}\left(3 L_{2} C_{2}-L_{2} C_{2} k^{2}-C_{2}^{2} R_{L}^{2}\right) \omega^{4}+L_{2}^{3} C_{2}^{3}\left(k^{2}-1\right) \omega^{6}\right)}{C_{2} L_{2} \omega\left(1+C_{2}\left(C_{2} R_{L}^{2}-2 L_{2}\right) \omega^{2}+\left(C_{2} L_{2}\right)^{2} \omega^{4}\right)}
\end{gathered}
$$

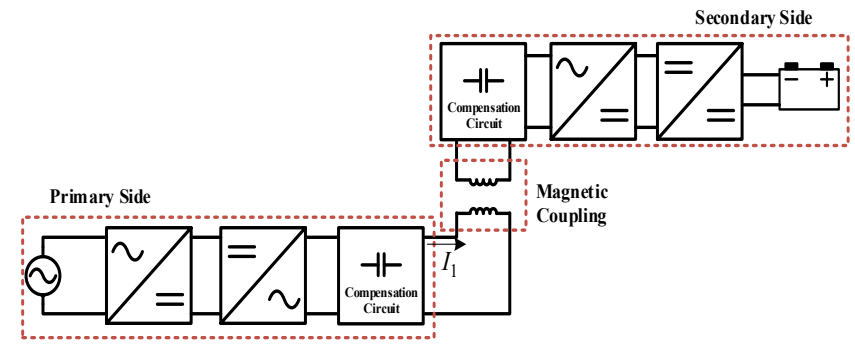

Fig. 1. The structure of WPT system.

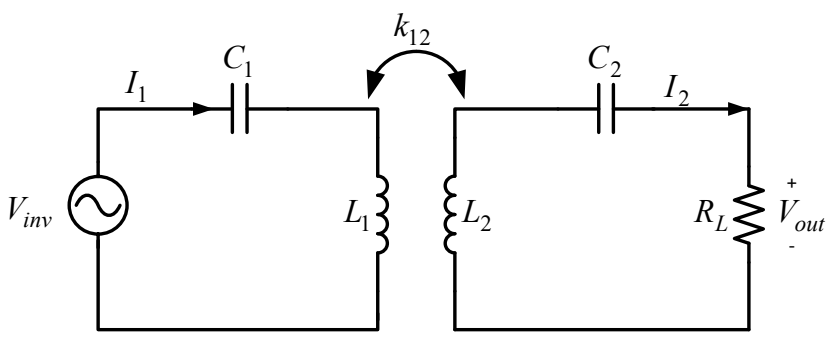

Fig. 2. The equivalent circuit of SS compensation WPT system.

\section{A. Impedance Analysis}

The input impedance $\left(Z_{\text {in }}\right)$ can exhibit inductive or capacitive behavior, depending on the switching frequency. Normally, the resonant inverter with a voltage source as the input is operated in the inductive region for turn-on ZVS operation [16]. The primary resonant circuit is intentionally tuned corresponding to the secondary poles in (2). The impedance $Z_{\text {in }}$ can exhibit inductive or capacitive behavior, depending on the switching frequency. The impedance $Z_{\text {in }}$ is given by (3) and (4).

Setting (4) to zero, the ZPA frequencies are obtained where the feasible solutions with positive frequency are given as $\omega_{0}, \omega_{1}$ and $\omega_{2}$. The natural frequency $\omega_{0}$ in (1) is one of the solutions that is not affected by the load and coupling variations. The other solutions, $\omega_{1}$ and $\omega_{2}$, are obtained as,

$\omega_{1}=\sqrt{\frac{C_{2}^{2}\left(R_{L}\right)^{2}-\frac{2}{\omega_{0}^{2}}-\sqrt{\frac{4}{\omega_{0}^{4}}\left(k_{12}^{2}-1\right)+\left[\frac{2}{\omega_{0}^{2}}-C_{2}^{2}\left(R_{L}\right)^{2}\right]^{2}}}{\frac{2}{\omega_{0}^{4}}\left(k_{12}^{2}-1\right)}}$ 


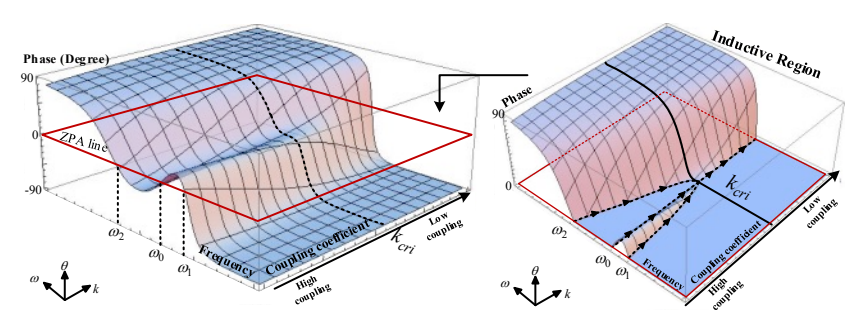

Fig. 3. The ZPA frequencies of SS compensation under coupling variations

It is noted that $\omega_{1}$ and $\omega_{2}$ can be both real and complex depending on the system parameters. The complex roots of $\omega_{1}$ and $\omega_{2}$ are only mathematically possible but not operable in practice. Only the real roots can give insightful meaning of the operating frequency as the ZPA frequency. The ZPA frequencies trajectories can be depicted in Fig. 3.

The different ZPA frequencies result in different impedances. The impedance at each ZPA frequency can be calculated by applying equations (1), (5) and (6) with (3). The impedances at the ZPA frequencies can be expressed as follows,

$$
\begin{gathered}
Z_{\text {in }}\left(\omega_{0}\right)=\frac{k_{12}^{2} L_{1}}{C_{2} R_{L}} \\
Z_{\text {in }}\left(\omega_{1}\right)=Z_{\text {in }}\left(\omega_{2}\right)=\frac{L_{1} R_{L}}{L_{2}}
\end{gathered}
$$

The input impedance at $\omega_{0}$ in (7) is dependent on the coupling and load. When the load resistance is decreased or the coupling is increased, the input impedance increases. On the other hand, the input impedance at $\omega_{1}$ and $\omega_{2}$ in (8) are readily independent with the coupling coefficient.

\section{B. Bifurcation Criteria}

As mentioned earlier, the usual reaction to the bifurcation phenomenon is to maintain the load resistance and coil coupling to avoid the bifurcation condition. Starting from a low coupling coefficient, there exists only one ZPA frequency at $\omega_{0}$. As the coupling coefficient is increased, $\omega_{I}$ and $\omega_{2}$ become real and the system is bifurcated with two additional ZPA frequencies. The relationships in (12) and (13) yield the bifurcated condition through the discriminants of (4). The bifurcation criteria coupling $k_{c r i}$ expressed as,

$$
k_{c r i}>\frac{R_{L}}{Z_{0}} \sqrt{1-\left(\frac{R_{L}}{2 Z_{0}}\right)^{2}}
$$

Or

$$
L_{2, c r i}<\frac{R_{L}^{4} C_{2}^{2}}{4\left(C_{2} R_{L}^{2} k_{12}^{2}\right)}
$$

where $Z_{0}$ is the characteristic impedance of the secondary resonant tank, expressed as,

$$
Z_{0}=\sqrt{\frac{L_{2}}{C_{2}}}
$$

The bifurcation criteria in (7) and (8) ensure the existence of $\omega_{1}$ and $\omega_{2}$. The $k_{c r i}$ represents an equivalent position of the secondary coil and the critical secondary coil inductance $\left(L_{2, c r i}\right)$ is the maximum inductance value that can be achieved the bifurcated condition.

\section{The Constant Current Gain under Coupling Independent Operation}

Referring to the input impedance in (3) and (4), the current and gain are given by

$$
\left|G_{I}(j \omega)\right|=\sqrt{\frac{L_{1} L_{2} C_{2}^{2} k_{12}^{2} \omega^{4}}{1-2 C_{2} L_{2} \omega^{2}+C_{2}^{2} \omega^{2}\left(\left(R_{L}\right)^{2}+L_{2}^{2} \omega^{2}\right)}}
$$

The current and voltage gains as a function of $\omega_{0}, \omega_{1}$ and $\omega_{2}$ can be calculated as follows,

$$
\begin{gathered}
\left|\frac{I_{2}}{I_{1}}\right|_{\omega=\omega_{0}}=\frac{k_{12}}{R_{L}} \sqrt{\frac{L_{1}}{C_{2}}} \\
\left|\frac{I_{2}}{I_{1}}\right|_{\omega=\omega_{1}}=\left|\frac{I_{2}}{I_{1}}\right|_{\omega=\omega_{2}}=\sqrt{\frac{L_{1}}{L_{2}}}
\end{gathered}
$$

The current gain in (11) is the gain at the frequency $\omega o$. The coupling coefficient $k$ plays a key role in current gain. Moreover, the input impedance in (7) is substantially reduced as the coupling coefficient $k$ decreases. If the frequency $\omega_{0}$ is chosen as the operating point, the maximum coupling coefficient must be taken into account for the targeted output power.

On the other hand, the existed frequencies $\omega_{1}$ and $\omega_{2}$ under bifurcation condition yield independent current and voltage gains from $k$. The gain in (14) effectively depend on the ratio between the primary and secondary inductances. Consequently, with the bifurcated operation, the WPT system behaves similar to an ideal transformer. The output power of the proposed system is given as,

$$
P_{\text {out }}\left(\omega_{2}\right)=\frac{L_{2} V_{\text {in }}^{2}}{L_{1} R_{L}}
$$

The output power of the proposed WPT system is independent from the coupling coefficient. With the bifurcated operation, the current gain and output power remain unchanged, whether the magnetic coupling is changed.

\section{Phase-Locked Loop Controller}

A phase-lock loop (PLL) controller is implemented to track the phase difference between the input voltage $\left(V_{i n}\right)$ and current $\left(I_{l}\right)$. For simplicity, all frequencies in $\mathrm{rad} / \mathrm{s}$ are changed to $\mathrm{Hz}$, for the rest of the paper. The PI controller adjusts the phase by regulating the frequency $f_{s}$ via the voltage-controlled-oscillator (VCO) circuit. The PLL controller starts at high frequency $\left(f_{s}>>f_{2}\right)$. Then, the frequency is reduced to find the ZPA frequency. During bifurcation, the PLL controller adjusts the switching 
frequency to slightly higher than $f_{2}$ to ensure ZVS operation. If the bifurcation condition is not met, the PLL controller tracks the frequency $f_{0}$ instead. The block diagram of the PLL controller, shown in Fig. 4.

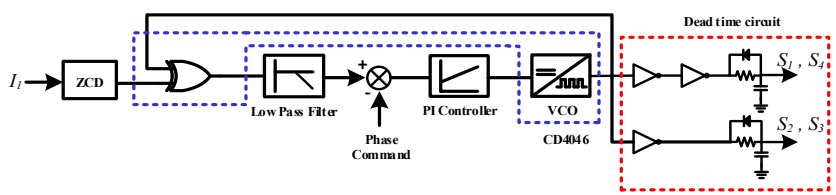

Fig. 4. The PLL Controller

\section{Simulation AND EXPERIMENTAL RESUlts}

In this work, two cases of common coil topologies are studied. The system in Case I is designed for the maximum vertical distance of $10 \mathrm{~cm}$. In Case II, the maximum vertical distance is at $20 \mathrm{~cm}$. The coil dimensions for both cases are given in Table 1 . The constructed coils are experimentally measured. The obtained parameters are shown in Table 2. Note that the maximum vertical distance of Cases I and II are constrained by the corresponding $k_{c r i}$ in which the bifurcation occurs.

TABLE I

COIL DIMENSIONS

\begin{tabular}{l|l|c|c}
\hline \hline \multirow{2}{*}{ Parameter } & \multicolumn{1}{|c|}{ DIMENSION } & CASE I & Case II \\
& Inside diameter (mm) & 120 & 340 \\
\multirow{3}{*}{$\begin{array}{l}\text { Primary } \\
\text { coil }\end{array}$} & Outside diameter (mm) & 280 & 530 \\
& Number of turns (N) & 17 & 19 \\
& Litz Wire & $200 *$ AWG31 & $200 *$ AWG31 \\
\cline { 2 - 4 } Secondary & Inside diameter (mm) & 120 & 180 \\
coil & Outside diameter (mm) & 280 & 395 \\
& Number of turns (N) & 17 & 19 \\
& Litz Wire & $200 *$ AWG31 & $200 *$ AWG31 \\
\hline \hline
\end{tabular}

TABLE II

SYSTEM PARAMETERS IN TWO EXPERIMENTAL CASES

\begin{tabular}{cccccc}
\hline \hline Parameter & Case I & Case II & Parameter & Case I & Case II \\
\hline$L_{I}(\mu \mathrm{H})$ & 68.6 & 209 & $f_{0}(\mathrm{kHz})$ & 42 & 44.1 \\
$L_{2}(\mu \mathrm{H})$ & 68.6 & 125 & $f_{I}(\mathrm{kHz})$ & 38.6 & 41 \\
$M(\mu \mathrm{H})$ & 20.6 & 35.6 & $f_{2}(\mathrm{kHz})$ & 49.5 & 49 \\
$k$ & 0.3 & 0.22 & $R_{c r i}(\Omega)$ & 7 & 10.9 \\
$C_{I}(\mathrm{nF})$ & 209 & 60 & $k_{c r i}$ & 0.27 & 0.144 \\
$C_{2}(\mathrm{nF})$ & 209 & 104 & $\mathrm{G}_{\mathrm{I}}$ & $\mathbf{1}$ & $\mathbf{1 . 3}$ \\
$R_{L}(\Omega)$ & 5 & 5 & $\mathrm{G}_{\mathrm{v}}$ & $\mathbf{0 . 9 2 6}$ & $\mathbf{0 . 7 2}$ \\
$R_{l}(\Omega)$ & 0.2 & 0.3 & $R_{2}(\Omega)$ & 0.2 & 0.2 \\
\hline \hline
\end{tabular}

\section{A. Frequency Response Simulation}

Frequency responses of the constructed WPT systems are obtained using the LTSPICE simulation software [17]. In Case I, the frequency response of the bifurcated system is shown in Fig. 5(a). Three ZPA frequencies are observed. At the resonant frequency $f_{0}$, the current $I_{2}$ is greater than $I_{l}$. The phase angle of $I_{2}$ is $270^{\circ}$ lagging or $90^{\circ}$ leading compared with $I_{l}$. When one current is at the maximum, the other is zero. At this stage, no active power is being transferred to the secondary circuit. At frequencies $f_{1}$ and $f_{2}$, the currents $I_{1}$ and $I_{2}$ are equal in magnitude. The phase difference between $I_{1}$ and $I_{2}$ is $180^{\circ}$ at the frequency $f_{1}$, whereas both currents are in phase at $f_{2}$. In other words, the WPT system exhibits an ideal transformer behavior at the operating frequencies $f_{1}$ and $f_{2}$, with different voltage polarities. The current gain is only related to the ratio between the primary and secondary inductances. Fig. 5(b) shows a similar frequency response of Case II. At the frequency $f_{0}$, the current $I_{2}$ is greater than $I_{1}$ and the phase angle of $I_{2}$ is leading by $90^{\circ}$. Both currents become greater at the frequencies $f_{1}$ and $f_{2}$. The currents $I_{1}$ and $I_{2}$ are $12.3 \mathrm{~A}$ and $16.2 \mathrm{~A}$, respectively with the current gain of 1.3 . At the frequency $f_{2}$, the phase difference between both currents is $360^{\circ}$.

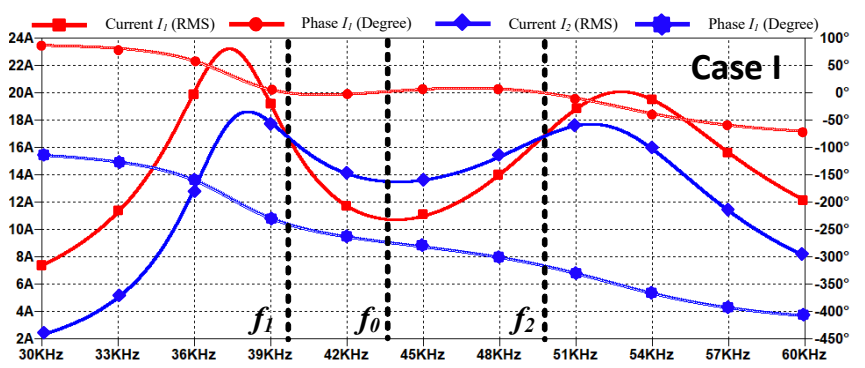

(a)

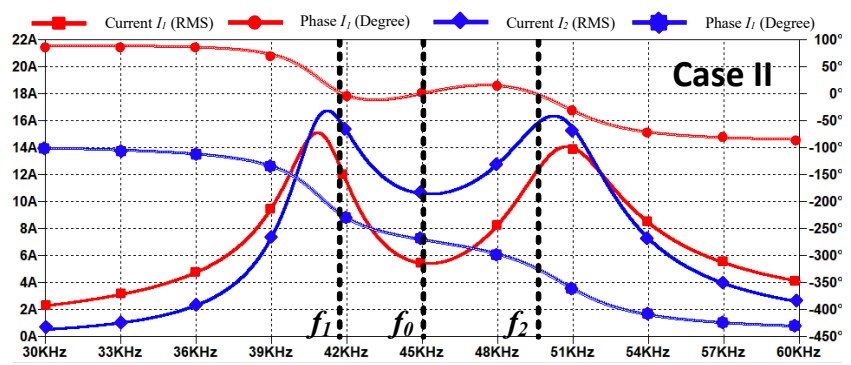

(b)

Fig. 5. The frequency response of the current $\mathrm{I}_{1}$ and $\mathrm{I}_{2}$, (a) Case $\mathrm{I}$, (b) Case II.

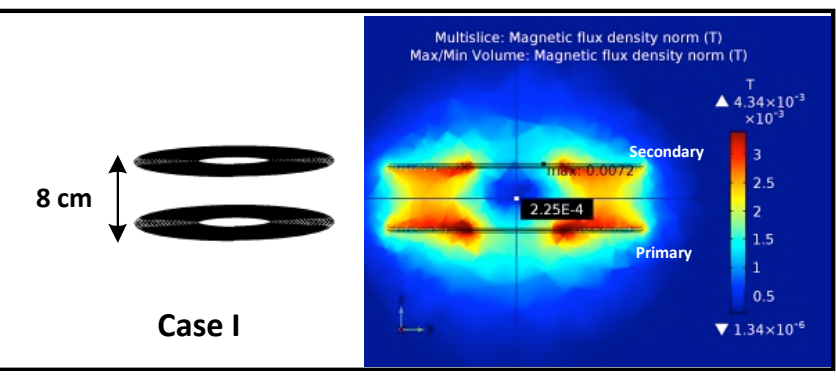

(a)

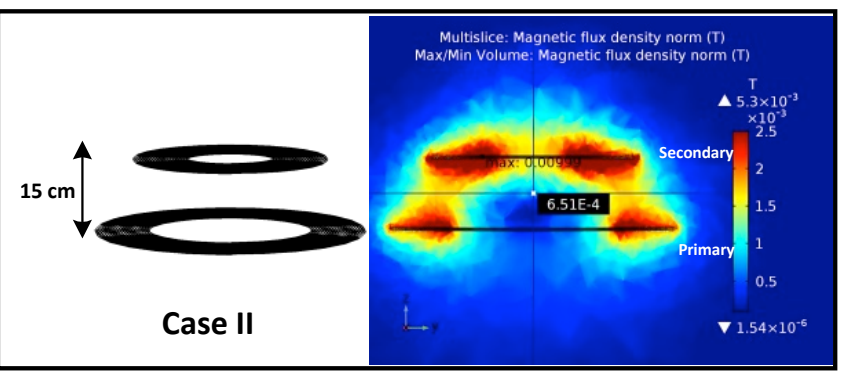

(b)

Fig. 6. Magnetic flux density in RMS value (a) Case I, (b) Case II 


\section{B. Finite Element Simulation}

The important benefit of the proposed work is that ferrite bar is no longer required since the voltage and current gains are independent from the coupling $k_{12}$. To illustrate the coupling independence, a finite element analysis (FEA) study for ferriteless primary and secondary coils is performed using the COMSOL Multiphysics software package [18]. The operating frequency is set to $f_{2}$. The magnetic flux density is measured along both primary and secondary coils, as shown in Figs. 6(a) and 6(b), for Case I and Case II, respectively. In Fig. 6(a), the maximum magnetic flux density at the secondary coil is at $7.2 \mathrm{mT}$, compared with $0.225 \mathrm{mT}$ at the middle point between both coils. This implies that the majority of the induced flux is the linkage flux and the leakage flux is negligible.

Similarly, the magnetic flux density of Case II is measured, as shown in Fig. 6(b). The maximum magnetic flux density at the secondary coil is $10 \mathrm{mT}$, compared with $0.65 \mathrm{mT}$ at the middle point between both coils. The linkage flux is 15.4 times over the leakage flux at the middle point between both coils.

\section{Experimental Waveforms}

Two WPT prototypes for cases I and II, are constructed with vertical distances of $10 \mathrm{~cm}$ and $15 \mathrm{~cm}$, respectively. The operation under the unity current gain is demonstrated through a symmetrical coil configuration in Case I. The higher current gain of 1.3 is represented through the asymmetrical configuration in Case II. Both systems can deliver $1 \mathrm{~kW}$ to the load. The system parameters are given in Table 3. The coupling coefficients for cases I and II are measured at 0.3 and 0.22 , respectively. Experimental waveforms are shown in Fig. 7 with the relevant measurement data listed in Table III. A $5-\Omega$ resistance is connected as the load. The PLL controller tracks the ZPA frequency $f_{2}$, and the inverter operates under ZVS mode with the phase angle of 8.8 degrees. The efficiencies of the systems in Cases I and II are 96\% and 93\%, respectively.

\section{Abruptly Misalignment Testing}

The asymmetrical configuration in Case II with the vertical distance of $10 \mathrm{~cm}$ is chosen to illustrate the system performance under a sudden movement of the secondary coil. Initially, the secondary coil is located in the perfect alignment position with the initial coupling $k$ of 0.3 . The secondary coil is then suddenly moved laterally to the horizontal position of $16 \mathrm{~cm}$. The coupling $k$ is reduced from 0.3 to 0.18 , well within the bifurcated condition. The switching frequency is reduced from $52.08 \mathrm{kHz}$ to 48.08 $\mathrm{kHz}$ to preserve the same current values of $I_{1}$ and $I_{2}$, as shown in Fig. 8. The current gain remains unchanged at 1.3. Since both currents are constant, the system output power and efficiency are constant under the sudden movement of the secondary coil. With the proposed operation, the loosely coupling WPT system exhibits similar behavior as an ironcore transformer. That is the current gain is independent from the coupling coefficient. This implies that the secondary current can be estimated from the primary current without a need for a current sensor on the secondary circuit, even under misaligned condition.

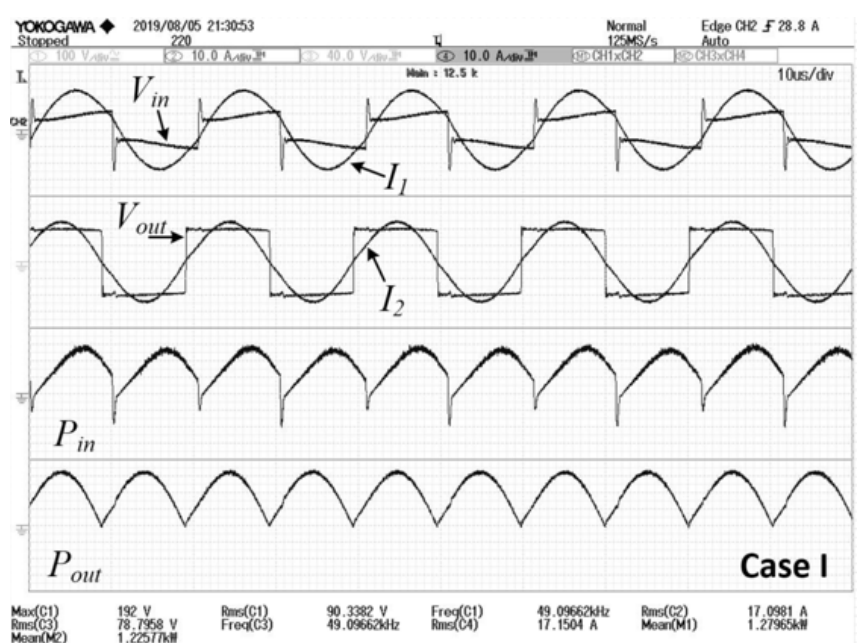

(a)

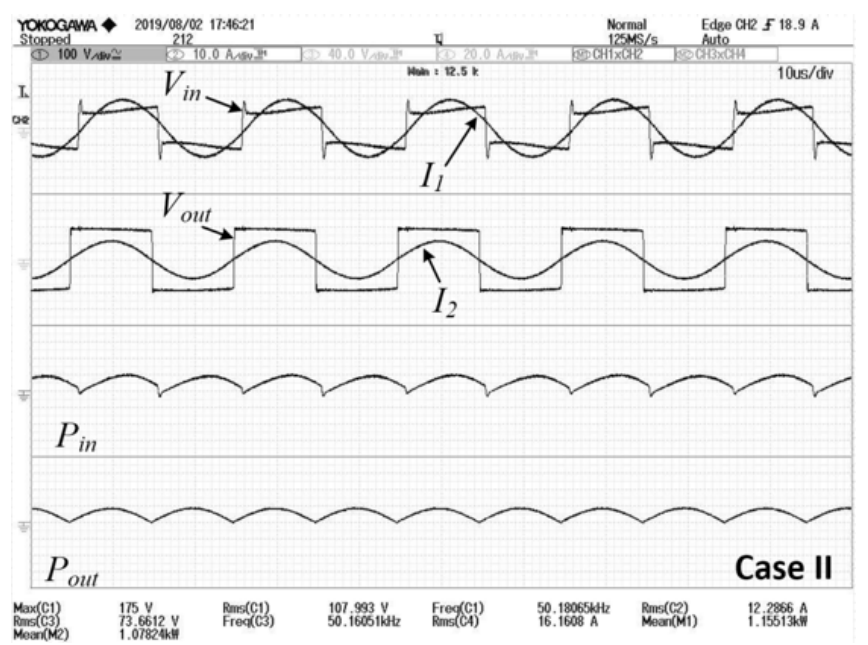

(b)

Fig. 7. The experimental waveforms in two cases. (Top to bottom trace) $\mathrm{V}_{\text {in }}$ Vs I $\mathrm{I}_{1}, \mathrm{~V}_{\text {out }}$ Vs I $\mathrm{I}_{2}, \mathrm{P}_{\text {in, }}, \mathrm{P}_{\text {out. }}$ (a) Case I, and (b) Case II.

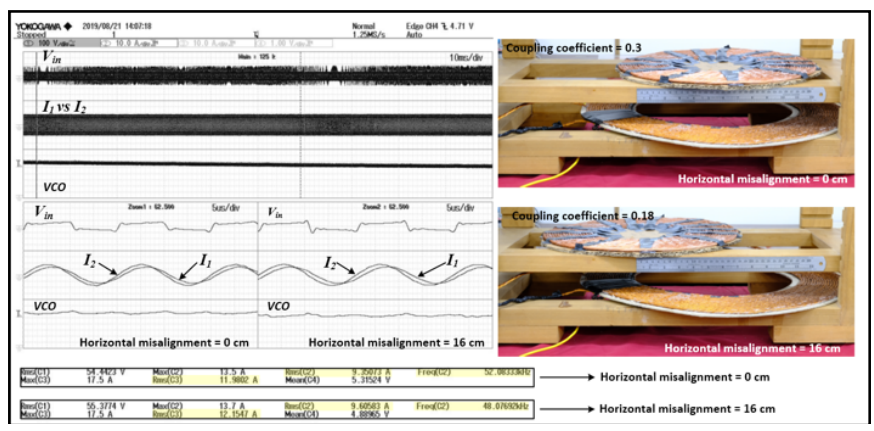

Fig. 8. The abruptly misalignment testing result

\section{CONCLUSION}

This paper proposes a new operation and control of a loosely coupling WPT system based on the previously unused operating regions due to the bifurcation phenomenon. The investigated mathematical model reveals feasible operating points with inherent constant current and power throughout the operation. The bifurcation criteria are adopted as the boundary condition. Since the current gain is independent from the coupling and load variation, the WPT 
system behave similar to an iron-core transformer with the benefit of secondary current estimation without a current sensor on the secondary circuit. The proposed method is validated by the created prototype with 2 coil configurations incorporated with the PLL controller. The FEA simulation results confirm that the majority of the induced flux is the linkage flux with relatively negligible leakage flux. The efficiency of the proposed system can reach $96 \%$ and $93 \%$ for Cases I and II, respectively.

\section{REFERENCES}

[1] C.-S. Wang, O. H. Stielau and G. A. Covic, "Design Considerations for a Contactless Electric Vehicle Battery Charger," IEEE Trans. Ind. Electron., vol. 52, no. 5, pp. 1308 - 1314, 2005.

[2] W. Zhang, S.-C. Wong, C. K. Tse and Q. Chen, "Analysis and Comparison of Secondary Series- and Parallel-Compensated Inductive Power Transfer Systems Operating for Optimal Efficiency and LoadIndependent Voltage-Transfer Ratio," IEEE Trans. Power Electron. vol. 29, no. 6, pp. 2979-2990, 2014.

[3] S. Samanta and A. K. Rathore, "Small-Signal Modelling and ClosedLoop Control of a Parallel-Series/Series Resonant Converter for Wireless Inductive Power Transfer," IEEE Trans. Ind. Electron., vol. 66, no. 1, pp. 172-182, 2019.

[4] B. Esteban, M. Sid - Ahmed and N. C. Kar, "A Comparative Study of Power Supply Architectures in Wireless EV Charging Systems," IEEE Trans. Power Electron., vol. 30, no. 11, pp. 6408 - 6422, 2015.

[5] C. Liu, S. Ge, Y. Guo, H. Li and G. Cai, "Double-LCL resonant compensation network for electric vehicles wireless power transfer: experimental study and analysis," IET Power Electron., vol. 9, no. 11, pp. 2262-2270, 2016.

[6] W. Zhang, S.-C. Wong, C. K. Tse and Q. Chen, "Design for Efficiency Optimization and Voltage Controllability of Series-Series Compensated Inductive Power Transfer Systems," IEEE Trans. Power Electron., vol. 29, no. 1, pp. 191-200, 2014.

[7] J. Jenson, J. P. Therattil and J. A. Johnson, "A Novel LCC-LCL Compensation WPT System for Better Performance," in IEEE International Conference on Electrical, Computer and Communication Technologies (ICECET), Coimbatore, India, 2019.

[8] M. Budhia, G. A. Covic and J. T. Boys, "Design and Optimization of Circular Magnetic Structures for Lumped Inductive Power Transfer System," IEEE Trans. Power Electron., vol. 26, no. 11, pp. 3096 3108, 2011.

[9] Y. Chen, R. Mai, Y. Zhang, M. Li and Z. He, "Improving Misalignment Tolerance for IPT system Using a Third-Coil," IEEE Trans. Power Electron., vol. 34, no. 4, pp. 3009-3013, 2019.

[10] Y. Li, J. Zhao, Q. Yang, L. Liu, J. Ma and X. Zhang, "A Novel Coil With High MIsalignment Tolerance for Wireless Power Transfer," IEEE Trans. Magn., vol. 55, no. 6, 2019.

[11] C.-S. Wang, G. Covic and O. Stielau, "Power Transfer Capability and Bifurcation Phenomena of Loosely Coupled Inductive Power Transfer Systems," IEEE Trans. Ind. Electron., vol. 51, no. 1, pp. 148-157, 2004.

[12] J. Boy, G. Covic and A. Green, "Stability and control of inductively coupled power transfer systems," Proc. IEE Electr. Power Appl., vol. 147, pp. 37-43, 2000.

[13] N.-C. Kuo, B. Zhao and A. M. Niknejad, "Bifurcation Analysis in Weakly-Coupled Inductive Power Transfer System," IEEE Trans. Circuits Syst. I, Reg. Papers, vol. 63, no. 5, pp. 727-738, 2016.

[14] K. Aditya and S. S. Williamson, "Design Guidelines to Avoid Bifurcation in a Series-Series Compensated Inductive Power Transfer System," IEEE Trans. Ind. Inform., vol. 66, no. 5, pp. 3973-3982, 2019.

[15] A. P. Sample, D. A. Meyer and J. R. Smith, "Analysis, Experimental Results, and Range Adaptation of Magnetically Coupled Resonators for Wireless Power Transfer," IEEE Trans. Ind. Inform., vol. 58, no. 2, pp. 544-554, 2011.

[16] N. Hatchavanich, A. Sangswang and M. Konghirun, "Operation Region of Novel Multi-Coil Series-Series Compensation in Wireless
Power Transfer System for Electric Vehicle Applications," in IEEE International Symposium on Circuits and System (ISCAS), Sapporo, 2019.

[17] "LTSPICE," Analog Devices, 2019. [Online]. Available: https://www.analog.com/en/design-center/design-tools-andcalculators/ltspice-simulator.html. [Accessed 2019 August 22].

[18] "Comsol Multiphysics," Comsol, 2019. [Online]. Available: https://www.comsol.com/support. [Accessed 22 August 2019]. 